\title{
Numerical Analysis of Time-Fractional Diffusion Equations via a Novel Approach
}

\author{
Nehad Ali Shah $\mathbb{D}^{1},{ }^{1}$ S. Saleem $\mathbb{D}^{2},{ }^{2}$ Ali Akgül, ${ }^{3}$ Kamsing Nonlaopon $\mathbb{D}^{4},{ }^{4}$ and Jae Dong Chung ${ }^{1}$ \\ ${ }^{1}$ Department of Mechanical Engineering, Sejong University, Seoul 05006, Republic of Korea \\ ${ }^{2}$ Department of Mathematics, College of Science, King Khalid University, Abha 61413, Saudi Arabia \\ ${ }^{3}$ Siirt University, Art and Science Faculty, Department of Mathematics, 56100 Siirt, Turkey \\ ${ }^{4}$ Department of Mathematics, Faculty of Science, Khon Kaen University, Khon Kaen 40002, Thailand
}

Correspondence should be addressed to Kamsing Nonlaopon; nkamsi@kku.ac.th

Received 15 March 2021; Revised 9 April 2021; Accepted 16 April 2021; Published 27 May 2021

Academic Editor: Qifeng Zhang

Copyright (C) 2021 Nehad Ali Shah et al. This is an open access article distributed under the Creative Commons Attribution License, which permits unrestricted use, distribution, and reproduction in any medium, provided the original work is properly cited.

\begin{abstract}
The aim of this paper is a new semianalytical technique called the variational iteration transform method for solving fractionalorder diffusion equations. In the variational iteration technique, identifying of the Lagrange multiplier is an essential rule, and variational theory is commonly used for this purpose. The current technique has the edge over other methods as it does not need extra parameters and polynomials. The validity of the proposed method is verified by considering some numerical problems. The solution achieved has shown that the better accuracy of the proposed technique. This paper proposes a simpler method to calculate the multiplier using the Shehu transformation, making a valuable technique to researchers dealing with various linear and nonlinear problems.
\end{abstract}

\section{Introduction}

In the last decade, significant achievements have been made to applying and the theory of fractional differential equations (FDEs). These problems are increasingly implemented to model equations in research fields as diverse as mechanical schemes, dynamical schemes, chaos, continuous-time random walks, control, chaos synchronization, subdiffusive systems and anomalous diffusive, wave propagation phenomena and unification of diffusion, and so on. The benefits of the fractional-order scheme are that it allows more significant degrees of freedom in the problem. An integer-order differential operator (DO) is a local operator, while a fractionalorder DO is a nonlocal operator, taking into account that a potential state depends not only on the current state but also on the past of all its previous instances. Fractional-order schemes have become famous for this valuable property. Another explanation for applying fractional-order derivatives is that they are naturally linked to memory structures that prevail in most physical and scientific structure models. The book by Spanier and Oldham [1] continued to play an essential role in the improvement of the subject. A few other primary results connected to the solution of FDEs can be identified in the books of Ross and Miller [2], Podlubny [3], and Kilbas et al. [4].

Adolf Fick introduces the laws of diffusion of Fick in 1885. After that, the second law of Fick became identified as the diffusion equation. Diffusion is the mesh atom's movement from a high chemical potential or higher concentration field to a lower concentration or low chemical potential field. Investigators have investigated classical wave and diffusion equations to many physical schemes, such as classical diffusion, slow diffusion, diffusion-wave combination, and classical wave equation. Many diffusion equation implementations, such as phase transformation, electrochemistry, magnetic fields, filtration, microbiology, acoustics, astrophysics, and biochemical group structures. Diffusion is determined by the gradient of the potential energy of the diffusing form. The gradient is the difference in the value of a number, e.g., concentration, strain, or temperature, with differences in one or more variables is often differentiated. Researchers have been seeking to recognize and reduce manufacturing systems problems to 
reach better productivity. In applied science schemes, there are various causes for entropy production. In heat engines, heat transfer, the primary source of entropy production is a mass transfer, the coupling between heat, entropy generation and chemical reaction, electrical conduction, as described in the seminal sequence of publications by Bejan et al. [5, 6]. Scholars have utilized different methods for the analysis of diffusion equations such as Chebyshev collocation technique [7], finite difference technique [8], finite volume element technique [9], variational iteration technique [10], two-step Adomian decomposition technique [11], finite volume technique [12], and Laplace transform [10].

In this paper, we implemented the variational iteration transform method to solve the fractional-order diffusion equations.

The fractional-order two-dimensional diffusion equation is given as

$$
\frac{\partial^{\delta} \mu}{\partial \eta^{\delta}}=\frac{\partial^{2} \mu}{\partial \zeta^{2}}+\frac{\partial^{2} \mu}{\partial \psi^{2}}, 0<\delta \leq 1, \eta \geq 0
$$

with initial condition

$$
\mu(\zeta, \psi, 0)=g(\zeta, \psi)
$$

The fractional-order three-dimensional diffusion equation

$$
\frac{\partial^{\delta} \mu}{\partial \eta^{\delta}}=\frac{\partial^{2} \mu}{\partial \zeta^{2}}+\frac{\partial^{2} \mu}{\partial \psi^{2}}+\frac{\partial^{2} \mu}{\partial \mathfrak{J}^{2}}, 0<\delta \leq 1, \eta \geq 0
$$

with initial condition

$$
\mu(\zeta, \psi, \mathfrak{J}, 0)=g(\zeta, \psi, \mathfrak{I})
$$

A Lagrange multiplier technique has been widely utilized to solve different types of nonlinear equations [13]. This occurs in mathematics and physics or certain related fields but has been established as a basic analytical method, i.e., a variational iteration method (VIM) to model differential equations [14]. The VIM was first recommended by $\mathrm{He}$ [15] and was implemented effectively to address the heat transformation problem [15-17]. Recently, several researchers have widely used this method to solve linear and nonlinear equations. The approach offers a consistent and efficient mechanism for a wide variety of applications in engineering and science. It is based on a specific Lagrange multiplier and has the merits of simplicity and easy implementation. Unlike conventional numerical approaches, VIM does not require linearization, discretion, or perturbation. The successive approximation provides quick convergence for the exact result [18-21]. The variational iteration method was introduced in 2010 using the modified Riemann-Liouville derivative [22]. Recently, a procedure combining in this sense Laplace transformation and VIM was proposed [23, 24], and Wu developed a modification via fractional calculus and Laplace transformation [25]. LVIM for solving nonlinear PDEs [26] and system of fractional PDEs [27].

\section{Basic Definitions}

2.1. Definition. The fractional-order Riemann-Liouville integral is defined as $[28,29]$

$$
I_{0}^{\delta} h(\eta)=\frac{1}{\Gamma(\delta)} \int_{0}^{\eta}(\eta-s)^{\delta-1} h(s) d s
$$

2.2. Definition. The fractional-order Caputo's derivative of $f(\eta)$ is given as $[28,29]$

$$
\begin{aligned}
D_{\eta}^{\delta} f(\eta) & =I^{J-\delta} f^{j}, J-1<\delta<J, J \in \mathbb{N} ; \\
\frac{d^{J}}{d \eta^{J}} h(\eta), \delta & =J, J \in \mathbb{N} .
\end{aligned}
$$

2.3. Definition. Shehu transformation is new and identical to other integral transformations defined for exponential order functions. In Set A, the function is defined by [30-32]

$$
A=\left\{v(\eta): \exists, \rho_{1}, \rho_{2}>0,|v(\eta)|<M e^{\frac{|\eta|}{\rho_{i}}} \text {, if } \eta \in[0, \infty)\right.
$$

The Shehu transform which is described as $S($.$) for a$ function $v(\eta)$ is defined as

$$
S\{v(\eta)\}=V(s, u)=\int_{0}^{\infty} e^{\frac{-s \eta}{u}} v(\eta) d \eta, \eta>0, s>0
$$

The Shehu transform of a function $\nu(\eta)$ is $V(s, \mu)$ : then, $v(\eta)$ is called the inverse of $V(s, \mu)$ which is given as

$$
S^{-1}\{V(s, u)\}=v(\eta) \text {, for } \eta \geq 0, S^{-1} \text { is inverse Shehu transform. }
$$

2.4. Definition. Shehu transform for nth derivatives is given as [30-32]

$$
S\left\{v^{(J)}(\eta)\right\}=\frac{s^{J}}{u^{J}} V(s, u)-\sum_{k=0}^{J-1}\left(\frac{s}{u}\right)^{J-k-1} v^{(k)}(0) .
$$

2.5. Definition. The fractional-order derivatives of Shehu transformation are defined as [30-32]

$S\left\{v^{(\delta)}(\eta)\right\}=\frac{s^{\delta}}{u^{\delta}} V(s, u)-\sum_{k=0}^{J-1}\left(\frac{s}{u}\right)^{\delta-k-1} v^{(k)}(0), 0<\beta \leq n$

2.6. Definition. The Mittag-Leffler function, $E_{\delta}(z)$ for $\delta>0$, is given as

$$
E_{\delta}(z)=\sum_{J=0}^{\infty} \frac{z^{J}}{\Gamma(\delta J+1)}, \delta>0, z \in \mathbb{C}
$$




\section{The Methodology of VITM}

This section introduces the general producer of VITM to solve time-fractional partial differential equation [23].

$$
D_{\eta}^{\delta} \mu(\zeta, \eta)+\mathscr{M}(\zeta, \eta)+\mathscr{N}(\zeta, \eta)-\mathscr{K}(\zeta, \eta)=0,0<\delta \leq 1
$$

with the initial sources

$$
\mu(\zeta, 0)=g_{1}(\zeta)
$$

where $D_{\eta}^{\delta}=\partial^{\delta} /\left(\partial \eta^{\delta}\right)$ is the fractional-order Caputo derivative and $\delta, \mathscr{M}$, and $\mathcal{N}$, are linear and nonlinear functions, respectively, and sources function $\mathscr{K}$.

The implementation of Shehu transformation to Eq. (13)

$$
S\left[D_{\eta}^{\delta} \mu(\zeta, \eta)\right]+S[\mathscr{M}(\zeta, \eta)+\mathscr{N}(\zeta, \eta)-\mathscr{K}(\zeta, \eta)]=0
$$

Using the differentiation property of Shehu transformation, we get

$S[\mu(\zeta, \eta)]-\left.\sum_{k=0}^{m-1} \frac{s^{\delta-k-1}}{u^{\delta-k}} \frac{\partial^{k} \mu(\zeta, \eta)}{\partial^{k} \eta}\right|_{\eta=0}=-S[\mathscr{M}(\zeta, \eta)+\mathscr{N}(\zeta, \eta)-\mathscr{K}(\zeta, \eta)]$

The Lagrange multiplier of the iterative system as

$$
\begin{aligned}
S\left[\mu_{m+1}(\zeta, \eta)\right]= & S\left[\mu_{m}(\zeta, \eta)\right]+\lambda(s)\left[\frac{s^{\delta}}{u^{\delta}} \mu_{m}(\zeta, \eta)-\left.\sum_{k=0}^{m-1} \frac{s^{\delta-k-1}}{u^{\delta-k}} \frac{\partial^{k} \mu(\zeta, \eta)}{\partial^{k} \eta}\right|_{\eta=0}\right. \\
& -S[\mathscr{K}(\zeta, \eta)]-S\{\mathscr{M}(\zeta, \eta)+\mathcal{N}(\zeta, \eta)\}] .
\end{aligned}
$$

A Lagrange multiplier as

$$
\lambda(s)=-\frac{u^{\delta}}{s^{\delta}} .
$$

Applying inverse Shehu transform $S^{-1}$, Eq. (17) can be defined as

$$
\begin{aligned}
\mu_{m+1}(\zeta, \eta)= & \mu_{m}(\zeta, \eta)-S^{-1}\left[\frac { u ^ { \delta } } { s ^ { \delta } } \left[\left.\sum_{k=0}^{m-1} \frac{s^{\delta-k-1}}{u^{\delta-k}} \frac{\partial^{k} \mu(\zeta, \eta)}{\partial^{k} \eta}\right|_{\eta=0}\right.\right. \\
& -S[\mathscr{K}(\zeta, \eta)]-S\{\mathscr{M}(\zeta, \eta)+\mathcal{N}(\zeta, \eta)\}]],
\end{aligned}
$$

the initial value can be described as

$$
\mu_{0}(\zeta, \eta)=S^{-1}\left[\frac{u^{\delta}}{s^{\delta}}\left\{\left.\sum_{k=0}^{m-1} \frac{s^{\delta-k-1}}{u^{\delta-k}} \frac{\partial^{k} \mu(\zeta, \eta)}{\partial^{k} \eta}\right|_{\eta=0}\right\}\right]
$$

\section{Implementation of VITM}

4.1. Problem. Consider the fractional-order diffusion equation [11]

$$
\frac{\partial^{\delta} \mu}{\partial \eta^{\delta}}=\frac{\partial^{2} \mu}{\partial \zeta^{2}}-\frac{\partial \mu}{\partial \zeta}+\mu \frac{\partial^{2} \mu}{\partial \zeta^{2}}-\mu^{2}+\mu 0<\alpha \leq 1,
$$

with the initial condition

$$
\mu(\zeta, 0)=e^{\zeta}
$$

Applying VITM on equation (21), we have

$$
\begin{aligned}
\mu_{m+1}(\zeta, \eta)= & S^{-1}\left[\frac{\mu_{m}(\zeta, \eta)}{s}\right]+S^{-1} \\
& \cdot\left[\lambda(s) S\left\{\frac{\partial^{2} \mu_{m}}{\partial \zeta^{2}}-\frac{\partial \mu_{m}}{\partial \zeta}+\mu_{m} \frac{\partial^{2} \mu_{m}}{\partial \zeta^{2}}-\mu_{m}^{2}+\mu_{m}\right\}\right],
\end{aligned}
$$

where the Lagrange multiplier is $\lambda(s)$

$$
\begin{aligned}
\lambda(s)= & -\frac{u^{\delta}}{s^{\Phi}}, \mu_{m}(\zeta, \eta) \\
\mu_{m+1}(\zeta, \eta)= & S^{1^{-1}}\left[\frac{S^{-1}}{s}\right] \\
& \cdot\left[\frac{u^{\delta}}{s^{\delta}} S\left\{\frac{\partial^{2} \mu_{m}}{\partial \zeta^{2}}-\frac{\partial \mu_{m}}{\partial \zeta}+\mu_{m} \frac{\partial^{2} \mu_{m}}{\partial \zeta^{2}}-\mu_{m}^{2}+\mu_{m}\right\}\right] .
\end{aligned}
$$

Now take,

$$
\mu_{0}(\zeta, \eta)=e^{\zeta}
$$

consequently, we get

$$
\begin{aligned}
& m=0,1,2,3 \cdots \\
& \mu_{1}(\zeta, \eta)=S^{-1}\left[\frac{\mu_{0}(\zeta, \eta)}{s}\right]-S^{-1}\left[\frac{u^{\delta}}{s^{\delta}} S\left\{\frac{\partial^{2} \mu_{0}}{\partial \zeta^{2}}-\frac{\partial \mu_{0}}{\partial \zeta}+\mu_{0} \frac{\partial^{2} \mu_{0}}{\partial \zeta^{2}}-\mu_{0}^{2}+\mu_{0}\right\}\right], \\
& \mu_{1}(\zeta, \eta)=e^{\zeta}+\frac{e^{\zeta} \eta^{\delta}}{\Gamma(\delta+1)}, \\
& \mu_{2}(\zeta, \eta)=S^{-1}\left[\frac{\mu_{1}(\zeta, \eta)}{s}\right]-S^{-1}\left[\frac{u^{\delta}}{s^{\delta}} S\left\{\frac{\partial^{2} \mu_{1}}{\partial \zeta^{2}}-\frac{\partial \mu_{1}}{\partial \zeta}+\mu_{1} \frac{\partial^{2} \mu_{1}}{\partial \zeta^{2}}-\mu_{1}^{2}+\mu_{1}\right\}\right], \\
& \mu_{2}(\zeta, \eta)=e^{\zeta}+\frac{e^{\zeta} \eta^{\delta}}{\Gamma(\delta+1)}+\frac{e^{\zeta} \eta^{2 \delta}}{\Gamma(2 \delta+1)}, \\
& \mu_{3}(\zeta, \eta)=S^{-1}\left[\frac{\mu_{2}(\zeta, \eta)}{s}\right]-S^{-1}\left[\frac{u^{\delta}}{s^{\delta}} S\left\{\frac{\partial^{2} \mu_{2}}{\partial \zeta^{2}}-\frac{\partial \mu_{2}}{\partial \zeta}+\mu_{2} \frac{\partial^{2} \mu_{2}}{\partial \zeta^{2}}-\mu_{2}^{2}+\mu_{2}\right\}\right], \\
& \mu_{3}(\zeta, \eta)=e^{\zeta}+\frac{e^{\zeta} \eta^{\delta}}{\Gamma(\delta+1)}+\frac{e^{\zeta} \eta^{2 \delta}}{\Gamma(2 \delta+1)}+\frac{e^{\zeta} \eta^{3 \delta}}{\Gamma(3 \delta+1)} .
\end{aligned}
$$


The approximate result of equation (21) can be achieved as

$$
\begin{aligned}
& \mu(\zeta, \eta)=e^{\zeta}+\frac{e^{\zeta} \eta^{\delta}}{\Gamma(\delta+1)}+\frac{e^{\zeta} \eta^{2 \delta}}{{ }_{n+1}(2 \delta+1)}+\frac{e^{\zeta} \eta^{3 \delta}}{\Gamma(3 \delta+1)}+\cdots+\frac{e^{\zeta} \eta^{m \delta}}{\Gamma(m \delta+1)}, \\
& \mu(\zeta, \eta)=e^{\zeta} \sum_{m=0}^{\infty} \frac{\left(\eta^{\delta}\right)}{\Gamma(m \delta+1)}=e^{\zeta} E_{\delta}\left(\eta^{\delta}\right) .
\end{aligned}
$$

The exact result of equation (21)

$$
\mu(\zeta, \eta)=e^{(\zeta+\eta)}
$$

In Figure 1, the exact and the VITM solutions of problem 1 at $\delta=1$ are show by subgraphs, respectively. From the given figures, it can be seen that both the VITM and exact results are in close contact with each other. Also, in Figure 2, the VITM results of problem 1 are investigated at different fractional-order $\delta=0.8$ and 0.6 of $3 \mathrm{D}$ and $2 \mathrm{D}$. It is analyzed that in Table 1, the time-fractional problem results are convergent to an integer order effect as time-fractional analysis to integer order.

4.2. Problem. Consider the two-dimensional fractional-order diffusion equation [11]

$$
\frac{\partial^{\delta} \mu}{\partial \eta^{\delta}}=\frac{\partial^{2} \mu}{\partial \zeta^{2}}+\frac{\partial^{2} \mu}{\partial \psi^{2}} 0<\delta \leq 1
$$

with the initial condition

$$
\mu(\zeta, \psi, 0)=(1-\psi) e^{\zeta}
$$

Applying VITM on equation (38), we have

$\mu_{m+1}(\zeta, \psi, \eta)=S^{-1}\left[\frac{\mu_{m}(\zeta, \psi, \eta)}{s}\right]+S^{-1}\left[\lambda(s) S\left\{\frac{\partial^{2} \mu_{m}}{\partial \zeta^{2}}+\frac{\partial^{2} \mu_{m}}{\partial \psi^{2}}\right\}\right]$,

where the Lagrange multiplier $\lambda(s)$ is

$$
\lambda(s)=-\frac{u^{\delta}}{s^{\delta}}
$$

$\mu_{m+1}(\zeta, \psi, \eta)=S^{-1}\left[\frac{\mu_{m}(\zeta, \psi, \eta)}{s}\right]-S^{-1}\left[\frac{u^{\delta}}{s^{\delta}} S\left\{\frac{\partial^{2} \mu_{m}}{\partial \zeta^{2}}+\frac{\partial^{2} \mu_{m}}{\partial \psi^{2}}\right\}\right]$.

Now take,

$$
\mu_{0}(\zeta, \psi, \eta)=(1-\psi) e^{\zeta}
$$

consequently, we get

$$
\begin{aligned}
m & =0,1,2,3 \cdots \\
\mu_{1}(\zeta, \psi, \eta) & =S^{-1}\left[\frac{\mu_{0}(\zeta, \psi, \eta)}{s}\right]-S^{-1}\left[\frac{u^{\delta}}{s^{\delta}} S\left\{\frac{\partial^{2} \mu_{0}}{\partial \zeta^{2}}+\frac{\partial^{2} \mu_{0}}{\partial \psi^{2}}\right\}\right],
\end{aligned}
$$

$$
\begin{aligned}
& \mu_{1}(\zeta, \psi, \eta)=(1-\psi) e^{\zeta}+(1-\psi) e^{\zeta} \frac{\eta^{\delta}}{\Gamma(\delta+1)}, \\
& \mu_{2}(\zeta, \psi, \eta)=S^{-1}\left[\frac{\mu_{1}(\zeta, \psi, \eta)}{s}\right]-S^{-1}\left[\frac{u^{\delta}}{s^{\delta}} S\left\{\frac{\partial^{2} \mu_{1}}{\partial \zeta^{2}}+\frac{\partial^{2} \mu_{1}}{\partial \psi^{2}}\right\}\right], \\
& \mu_{2}(\zeta, \psi, \eta)=(1-\psi) e^{\zeta}+(1-\psi) e^{\zeta} \frac{\eta^{\delta}}{\Gamma(\delta+1)}+(1-\psi) e^{\zeta} \frac{\eta^{2 \delta}}{\Gamma(2 \delta+1)}, \\
& \mu_{3}(\zeta, \psi, \eta)=S^{-1}\left[\frac{\mu_{2}(\zeta, \psi, \eta)}{s}\right]-S^{-1}\left[\frac{u^{\delta}}{s^{\delta}} S\left\{\frac{\partial^{2} \mu_{2}}{\partial \zeta^{2}}+\frac{\partial^{2} \mu_{2}}{\partial \psi^{2}}\right\}\right], \\
& \mu_{3}(\zeta, \psi, \eta)=(1-\psi) e^{\zeta}+(1-\psi) e^{\zeta} \frac{\eta^{\delta}}{\Gamma(\delta+1)}+(1-\psi) e^{\zeta} \frac{\eta^{2 \delta}}{\Gamma(2 \delta+1)} \\
&+(1-\psi) e^{\zeta} \frac{\eta^{3 \delta}}{\Gamma(3 \delta+1)} .
\end{aligned}
$$

The approximate result of equation (38) can be achieved as

$$
\begin{aligned}
\mu(\zeta, \psi, \eta)= & (1-\psi) e^{\zeta}+(1-\psi) e^{\zeta} \frac{\eta^{\delta}}{\Gamma(\delta+1)}+(1-\psi) e^{\zeta} \frac{\eta^{2 \delta}}{\Gamma(2 \delta+1)} \\
& +(1-\psi) e^{\zeta} \frac{\eta^{3 \delta}}{\Gamma(3 \delta+1)}+\cdots+(1-\psi) e^{\zeta} \frac{\eta^{m \delta}}{\Gamma(m \delta+1)} .
\end{aligned}
$$

When $\alpha=1$, the VITM solution is

$$
\mu(\zeta, \psi, \eta)=(1-\psi) e^{\zeta} \sum_{m=0}^{\infty} \frac{(\eta)^{m}}{m !}
$$

The exact solution in closed form is

$$
\mu(\zeta, \psi, \eta)=(1-\psi) e^{(\zeta+\eta)}
$$

In Figure 3, the exact and the VITM solutions of problem 2 at $\delta=1$ are shown by subgraphs, respectively. From the given figures, it can be seen that both the VITM and exact results are in close contact with each other. Also, in Figure 4, the VITM results of problem 2 are investigated at different fractional-order $\delta=0.8$ and 0.6 of 3D and 2D. It is analyzed that in Table 2, the time-fractional problem results are convergent to an integer order effect as time-fractional analysis to integer order.

4.3. Problem. Consider the two-dimensional fractional-order diffusion equation [11]

$$
\frac{\partial^{\delta} \mu}{\partial \eta^{\delta}}=\frac{\partial^{2} \mu}{\partial \zeta^{2}}+\frac{\partial^{2} \mu}{\partial \psi^{2}} 0<\delta \leq 1
$$

with the initial condition

$$
\mu(\zeta, \psi, 0)=e^{(\zeta+\psi)}
$$



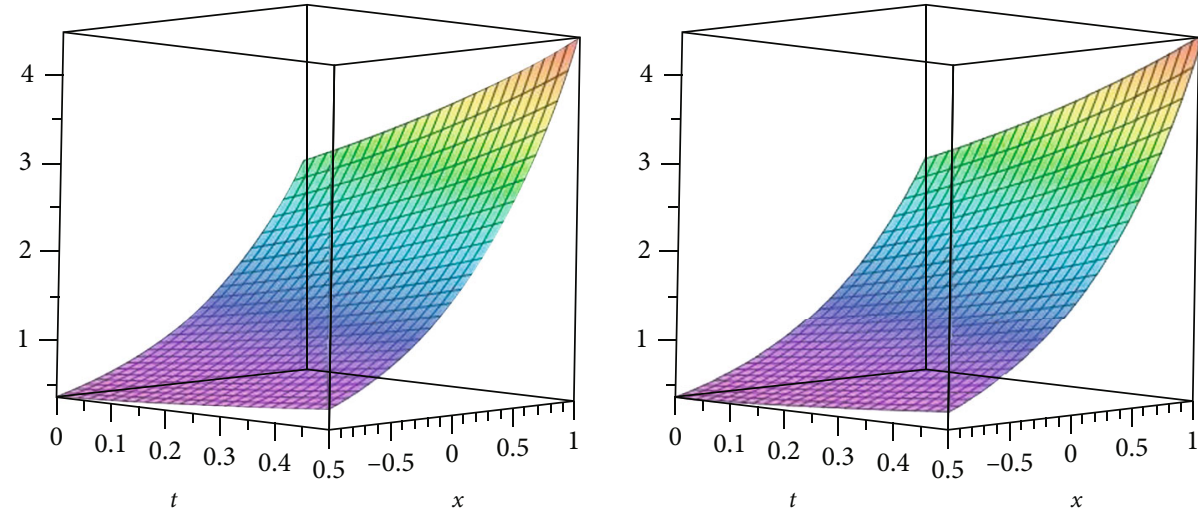

Figure 1: Graph of exact and analytical results of Problem 3.1.
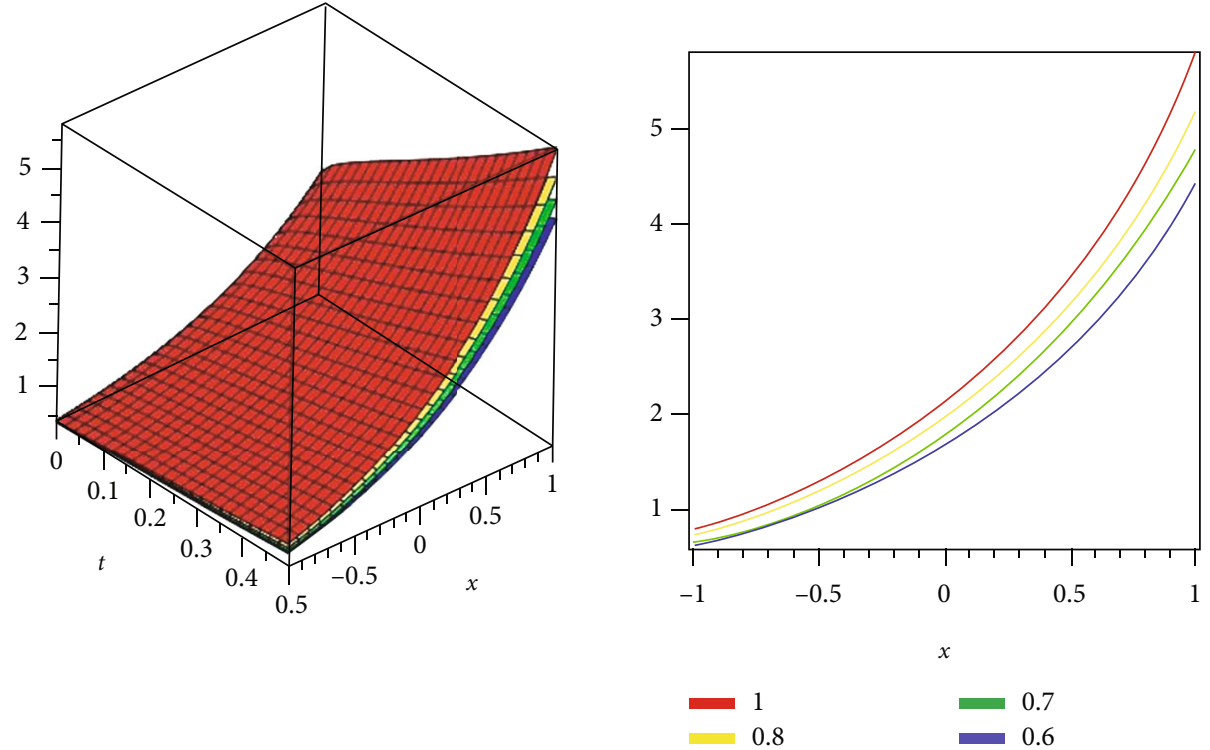

FIgURe 2: The different fractional-order graphs of Problem 3.1.

Applying VITM on equation (38), we have

$$
\mu_{m+1}(\zeta, \psi, \eta)=S^{-1}\left[\frac{\mu_{m}(\zeta, \psi, \eta)}{s}\right]+S^{-1}\left[\lambda(s) S\left\{\frac{\partial^{2} \mu_{m}}{\partial \zeta^{2}}+\frac{\partial^{2} \mu_{m}}{\partial \psi^{2}}\right\}\right],
$$

where the Lagrange multiplier $\lambda(s)$ is

$$
\begin{aligned}
\lambda(s) & =-\frac{u^{\delta}}{s^{\delta}} \\
\mu_{m+1}(\zeta, \psi, \eta) & =S^{-1}\left[\frac{\mu_{m}(\zeta, \psi, \eta)}{s}\right]-S^{-1}\left[\frac{u^{\delta}}{s^{\delta}} S\left\{\frac{\partial^{2} \mu_{m}}{\partial \zeta^{2}}+\frac{\partial^{2} \mu_{m}}{\partial \psi^{2}}\right\}\right] .
\end{aligned}
$$

Now take,

$$
\mu_{0}(\zeta, \psi, \eta)=e^{(\zeta+\psi)}
$$

consequently, we get

$$
\begin{gathered}
m=0,1,2,3 \cdots \\
\mu_{1}(\zeta, \psi, \eta)=S^{-1}\left[\frac{\mu_{0}(\zeta, \psi, \eta)}{s}\right]-S^{-1}\left[\frac{u^{\delta}}{s^{\delta}} s\left\{\frac{\partial^{2} \mu_{0}}{\partial \zeta^{2}}+\frac{\partial^{2} \mu_{0}}{\partial \psi^{2}}\right\}\right], \\
\mu_{1}(\zeta, \psi, \eta)=e^{(\zeta+\psi)}+2 e^{(\zeta+\psi)} \frac{\eta^{\delta}}{\Gamma(\delta+1)}, \\
\mu_{2}(\zeta, \psi, \eta)=S^{-1}\left[\frac{\mu_{1}(\zeta, \psi, \eta)}{s}\right]-S^{-1}\left[\frac{u^{\delta}}{s^{\delta}} s\left\{\frac{\partial^{2} \mu_{1}}{\partial \zeta^{2}}+\frac{\partial^{2} \mu_{1}}{\partial \psi^{2}}\right\}\right], \\
\mu_{2}(\zeta, \psi, \eta)=e^{(\zeta+\psi)}+2 e^{(\zeta+\psi)} \frac{\eta^{\delta}}{\Gamma(\delta+1)}+4 e^{(\zeta+\psi)} \frac{\eta^{2 \delta}}{\Gamma(2 \delta+1)}, \\
\mu_{3}(\zeta, \psi, \eta)=S^{-1}\left[\frac{\mu_{2}(\zeta, \psi, \eta)}{s}\right]-S^{-1}\left[\frac{u^{\delta}}{s^{\delta}} S\left\{\frac{\partial^{2} \mu_{2}}{\partial \zeta^{2}}+\frac{\partial^{2} \mu_{2}}{\partial \psi^{2}}\right\}\right], \\
\mu_{3}(\zeta, \psi, \eta)=e^{(\zeta+\psi)}+2 e^{(\zeta+\psi)} \frac{\eta^{\delta}}{\Gamma(\delta+1)}+4 e^{(\zeta+\psi)} \frac{\eta^{2 \delta}}{\Gamma(2 \delta+1)} \\
+16 e^{(\zeta+\psi)} \frac{\eta^{3 \delta}}{\Gamma(3 \delta+1)},
\end{gathered}
$$


TABLe 1: VITM at fractional-order value $\delta=0.8$ and absolute error $\delta=1$ of example 1 .

\begin{tabular}{|c|c|c|c|c|c|}
\hline$\eta$ & $\zeta$ & $\delta=0.75$ & $\delta=1$ & Exact & $\operatorname{AE}(\delta=1)$ \\
\hline \multirow{5}{*}{0.5} & 1 & 0.853687662 & 0.7165300518 & 0.7165306597 & $7.56 \mathrm{E}-08$ \\
\hline & 2 & 0.383587401 & 0.3331299364 & 0.3331301601 & 3.37E-08 \\
\hline & 3 & 0.210647180 & 0.0930849163 & 0.0930849986 & 9.35E-09 \\
\hline & 4 & 0.048026028 & 0.0411973531 & 0.0411973834 & 4.24E-09 \\
\hline & 5 & 0.024621114 & 0.0222089854 & 0.0222089965 & $2.10 \mathrm{E}-09$ \\
\hline \multirow{5}{*}{1} & 1 & 1.389724464 & 0.9988367591 & 1.0000000000 & $9.35 \mathrm{E}-06$ \\
\hline & 2 & 0.580784320 & 0.4778488184 & 0.4778794412 & $4.42 \mathrm{E}-06$ \\
\hline & 3 & 0.283191872 & 0.2453240178 & 0.2453352832 & $2.24 \mathrm{E}-06$ \\
\hline & 4 & 0.074713729 & 0.0587829240 & 0.0587870683 & $5.45 \mathrm{E}-07$ \\
\hline & 5 & 0.034438971 & 0.0193141142 & 0.0193156388 & 2.32E-07 \\
\hline
\end{tabular}
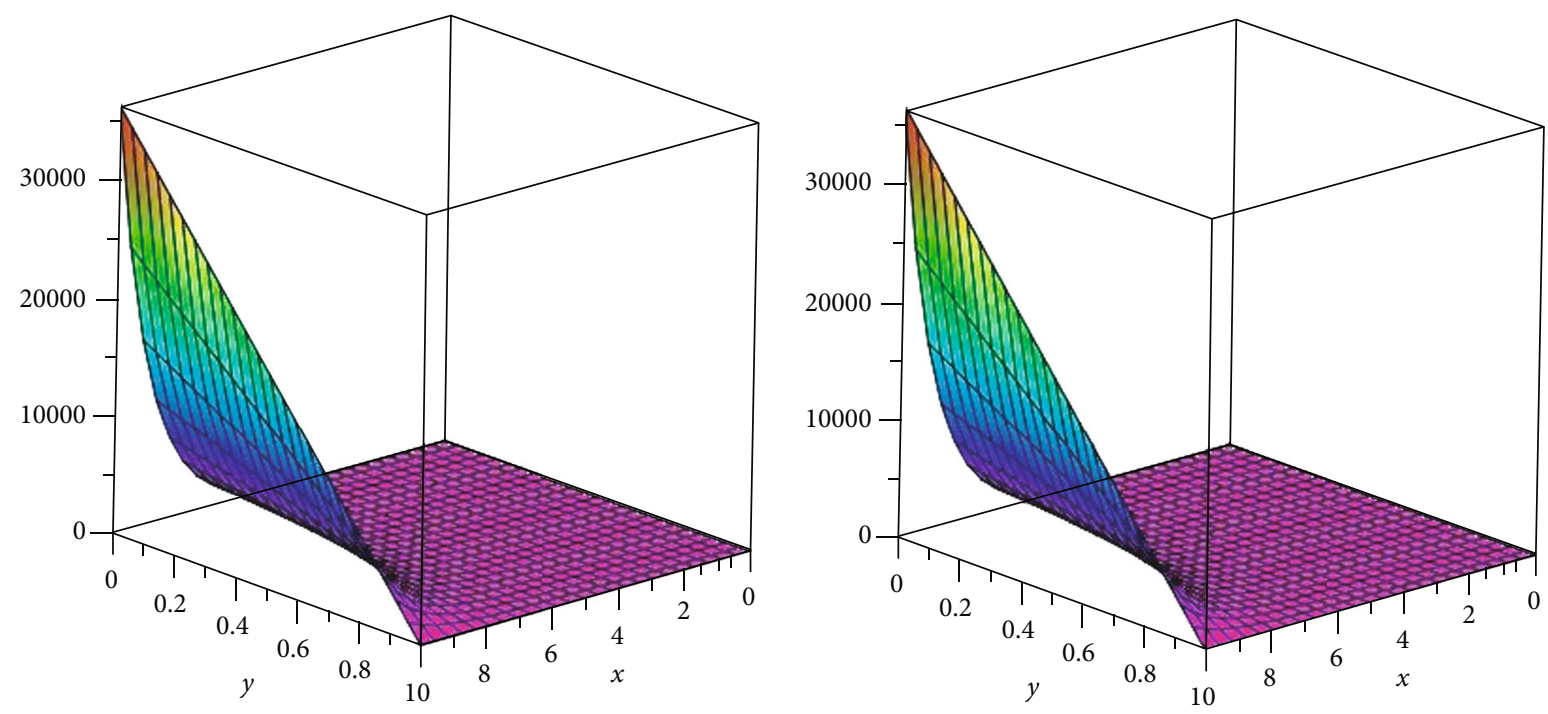

Figure 3: Graph of exact and approximate solutions of Problem 3.2.

The approximate result of equation (38) can be achieved as

$$
\begin{aligned}
\mu(\zeta, \psi, \eta)= & e^{(\zeta+\psi)}+2 e^{(\zeta+\psi)} \frac{\eta^{\delta}}{\Gamma(\delta+1)}+4 e^{(\zeta+\psi)} \frac{\eta^{2 \delta}}{\Gamma(2 \delta+1)} \\
& +16 e^{(\zeta+\psi)} \frac{\eta^{3 \delta}}{\Gamma(3 \delta+1)}+\cdots+(2)^{m} e^{(\zeta+\psi)} \frac{\eta^{m \delta}}{\Gamma(m \delta+1)} \\
\mu(\zeta, \psi, \eta)= & e^{(\zeta+\psi)}\left(1+\frac{2 \eta^{\delta}}{\Gamma(\delta+1)}+\frac{\left(2 \eta^{\delta}\right)^{2}}{\Gamma(2 \delta+1)}+\frac{\left(2 \eta^{\delta}\right)^{3}}{\Gamma(3 \delta+1)}\right. \\
& \left.+\frac{\left(2 \eta^{\delta}\right)^{4}}{\Gamma(4 \delta+1)}+\cdots+\frac{\left(2 \eta^{\delta}\right)^{m}}{\Gamma(m \delta+1)}\right) \\
\mu(\zeta, \psi, \eta)= & e^{(\zeta+\psi)} \sum_{m=0}^{\infty} \frac{\left(\eta^{\delta}\right)^{m}}{\Gamma(m \delta+1)}=(1-\psi) e^{\zeta} E_{\delta}\left(\eta^{\delta}\right) .
\end{aligned}
$$

When $\delta=1$, then the VITM solution is

$$
\mu(\zeta, \psi, \eta)=e^{(\zeta+\psi)} \sum_{m=0}^{\infty} \frac{(\eta)^{m}}{m !}
$$

The exact solution in closed form is

$$
\mu(\zeta, \psi, \eta)=e^{(\zeta+\psi+\eta)}
$$

In Figure 5, the exact and the VITM solutions of problem 3 at $\delta=1$ are shown by subgraphs, respectively. From the given figures, it can be seen that both the VITM and exact results are in close contact with each other. Also, in Figure 6, the VITM results of problem 3 are investigated at different fractional-order $\delta=0.8$ and 0.6 of $3 \mathrm{D}$ and $2 \mathrm{D}$. It is analyzed that time-fractional problem results are convergent to an integer order effect as time-fractional analysis to integer order.

4.4. Problem. Consider the three-dimensional fractionalorder diffusion equation [11]

$$
\frac{\partial^{\delta} \mu}{\partial \eta^{\delta}}=\frac{\partial^{2} \mu}{\partial \zeta^{2}}+\frac{\partial^{2} \mu}{\partial \psi^{2}}+\frac{\partial^{2} \mu}{\partial \mathfrak{J}^{2}}, 0<\delta \leq 1, \eta \geq 0
$$



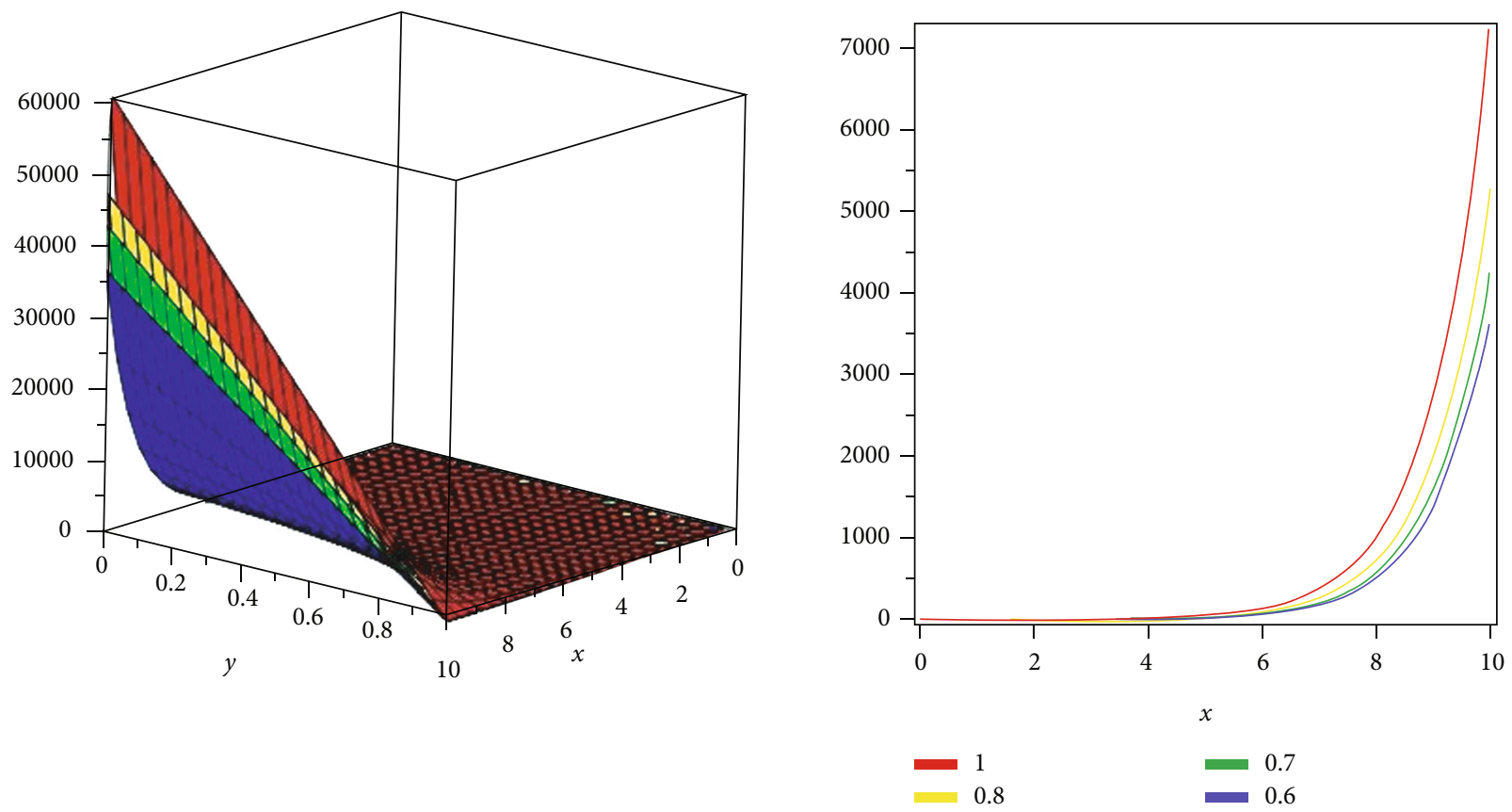

Figure 4: The different fractional-order $\delta$ graphs of Problem 3.2.

TABLE 2: VITM at fractional-order value $\delta=0.8$ and absolute error $\delta=1$ of example 2 .

\begin{tabular}{cccccc}
\hline$\eta$ & $\zeta$ & $\delta=0.75$ & $\delta=1$ & Exact & AE $(\delta=1)$ \\
\hline & 1 & 0.6873975264 & 0.4262266609 & 0.326227766 & 0.042622776 \\
0.5 & 0.0687397526 & 0.0426226660 & 0.004262277 & $2.34 \mathrm{E}-08$ \\
& 2 & 0.0067739752 & 0.0042622666 & 0.000436227 & $0.21 \mathrm{E}-09$ \\
& 3 & 0.0006773975 & 0.0004362266 & 0.000044622 & $2.14 \mathrm{E}-10$ \\
& 4 & 0.0000687397 & 0.0000446226 & 0.100000000 & $4.56 \mathrm{E}-05$ \\
& 5 & 2.7771190120 & 0.9984599728 & 0.010000000 & $7.89 \mathrm{E}-07$ \\
1 & 1 & 0.3567119012 & 0.0998953372 & 0.001000000 & $7.89 \mathrm{E}-08$ \\
& 2 & 0.0376711901 & 0.0098746437 & 0.000100000 & $7.22 \mathrm{E}-09$ \\
\hline
\end{tabular}

with the initial condition

$$
\mu(\zeta, \psi, \mathfrak{J}, 0)=\sin \zeta \sin \psi \sin \mathfrak{\Im}
$$

Applying VITM on equation (47), we have

$$
\begin{aligned}
\mu_{m+1}(\zeta, \psi, \mathfrak{J}, \eta)= & S^{-1}\left[\frac{\mu_{m}(\zeta, \psi, \mathfrak{J}, \eta)}{s}\right] \\
& +S^{-1}\left[\lambda(s) S\left\{\frac{\partial^{2} \mu_{m}}{\partial \zeta^{2}}+\frac{\partial^{2} \mu_{m}}{\partial \psi^{2}}+\frac{\partial^{2} \mu_{m}}{\partial \mathfrak{J}^{2}}\right\}\right],
\end{aligned}
$$

where the Lagrange multiplier $\lambda(s)$ is

$$
\begin{aligned}
\lambda(s)= & -\frac{u^{\delta}}{s^{\delta}} \\
\mu_{m+1}(\zeta, \psi, \mathfrak{J}, \eta)= & S^{-1}\left[\frac{\mu_{m}(\zeta, \psi, \mathfrak{J}, \eta)}{s}\right] \\
& -S^{-1}\left[\frac{u^{\delta}}{s^{\delta}} S\left\{\frac{\partial^{2} \mu_{m}}{\partial \zeta^{2}}+\frac{\partial^{2} \mu_{m}}{\partial \psi^{2}}+\frac{\partial^{2} \mu_{m}}{\partial \mathfrak{J}^{2}}\right\}\right] .
\end{aligned}
$$

Now take,

$$
\mu_{0}(\zeta, \psi, \mathfrak{J}, \eta)=\sin \zeta \sin \psi \sin \mathfrak{\Im}
$$



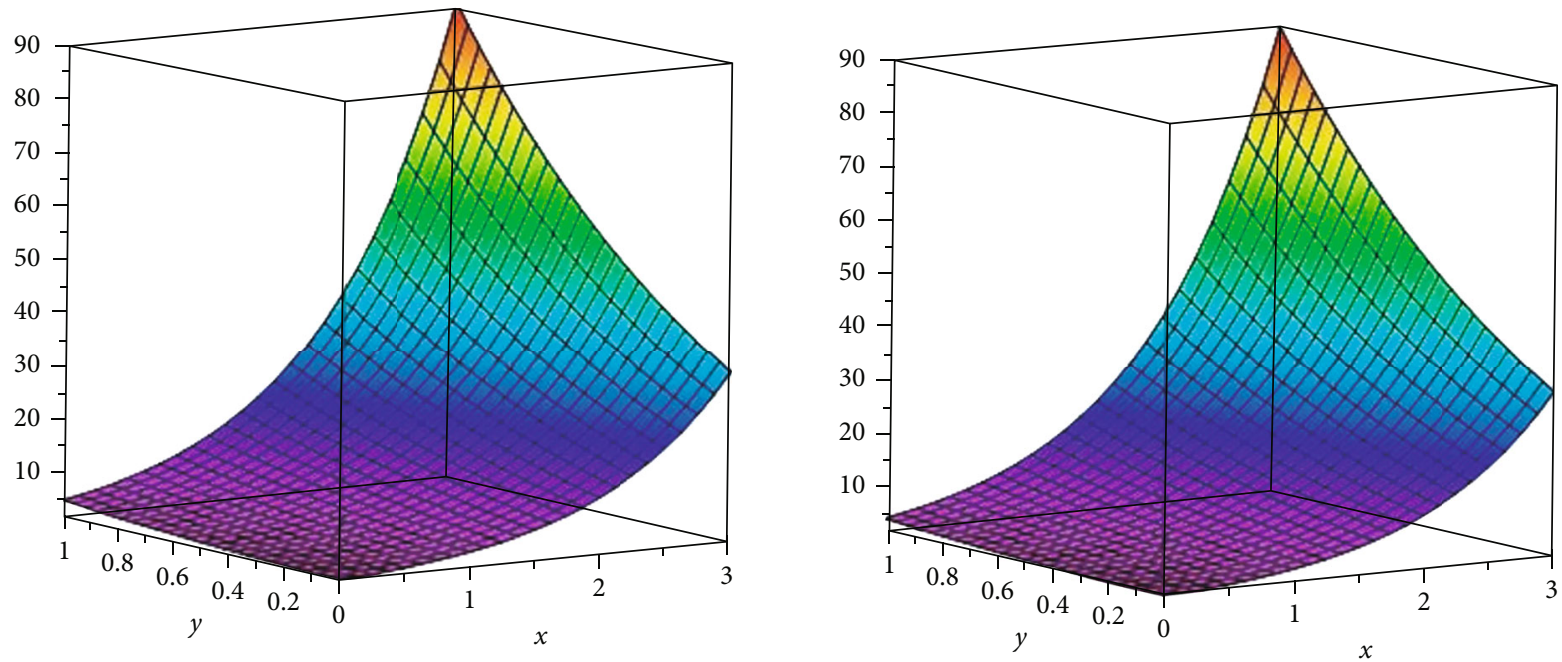

Figure 5: Graph of exact and approximate solutions of Problem 3.3.

consequently, we get

$$
\begin{aligned}
m= & 0,1,2,3 \cdots \\
\mu_{1}(\zeta, \psi, \mathfrak{I}, \eta)= & S^{-1}\left[\frac{\mu_{0}(\zeta, \psi, \mathfrak{\Im}, \eta)}{s}\right] \\
& \left.-S^{-1}\left[\frac{u^{\delta}}{s^{\delta}} S \frac{\partial^{2} \mu_{0}}{\partial \zeta^{2}}+\frac{\partial^{2} \mu_{0}}{\partial \psi^{2}}+\frac{\partial^{2} \mu_{0}}{\partial \mathfrak{J}^{2}}\right\}\right], \\
\mu_{1}(\zeta, \psi, \mathfrak{J}, \eta)= & \sin \zeta \sin \psi \sin \mathfrak{\Im}\left(1-3 \frac{\eta^{\delta}}{\Gamma(\delta+1)}\right), \\
\mu_{2}(\zeta, \psi, \mathfrak{J}, \eta)= & S^{-1}\left[\frac{\mu_{1}(\zeta, \psi, \mathfrak{J}, \eta)}{s}\right] \\
& \left.-S^{-1}\left[\frac{u^{\delta}}{s^{\delta}} S \frac{\partial^{2} \mu_{1}}{\partial \zeta^{2}}+\frac{\partial^{2} \mu_{1}}{\partial \psi^{2}}+\frac{\partial^{2} \mu_{1}}{\partial \mathfrak{J}^{2}}\right\}\right],
\end{aligned}
$$

$\mu_{2}(\zeta, \psi, \Im, \eta)=\sin \zeta \sin \psi \sin \mathfrak{\Im}\left(1-3 \frac{\eta^{\delta}}{\Gamma(\delta+1)}\right.$

$$
\begin{aligned}
& \left.+(-3)^{2} \frac{\eta^{2 \delta}}{\Gamma(2 \delta+1)}\right), \\
\mu_{3}(\zeta, \psi, \mathfrak{I}, \eta)= & S^{-1}\left[\frac{\mu_{2}(\zeta, \psi, \mathfrak{J}, \eta)}{s}\right] \\
& -S^{-1}\left[\frac{u^{\delta}}{s^{\delta}} S\left\{\frac{\partial^{2} \mu_{2}}{\partial \zeta^{2}}+\frac{\partial^{2} \mu_{2}}{\partial \psi^{2}}+\frac{\partial^{2} \mu_{2}}{\partial \mathfrak{J}^{2}}\right\}\right],
\end{aligned}
$$

$\mu_{3}(\zeta, \psi, \mathfrak{J}, \eta)=\sin \zeta \sin \psi \sin \Im\left(1-3 \frac{\eta^{\delta}}{\Gamma(\delta+1)}\right.$

$$
\left.+(-3)^{2} \frac{\eta^{2 \delta}}{\Gamma(2 \delta+1)}+(-3)^{3} \frac{\eta^{3 \delta}}{\Gamma(3 \delta+1)}\right)
$$

The approximate result of equation (47) can be achieved as $u(\zeta, \psi, \mathfrak{I}, \eta)=\sin \zeta \sin \psi \sin \mathfrak{I}$

$$
\begin{aligned}
& \cdot\left(1-3 \frac{\eta^{\delta}}{\Gamma(\delta+1)}+(-3)^{2} \frac{\eta^{2 \delta}}{\Gamma(2 \delta+1)}\right. \\
& \left.+(-3)^{3} \frac{\eta^{3 \delta}}{\Gamma(3 \delta+1)}+\cdots+(-3)^{m} \frac{\eta^{m \delta}}{\Gamma(m \delta+1)}\right) .
\end{aligned}
$$

When $\delta=1$, then the VITM solution in a closed form is

$$
\begin{aligned}
\mu(\zeta, \psi, \mathfrak{\Im}, \eta)= & \sin \zeta \sin \psi \sin \mathfrak{\Im} \\
& \cdot\left(1-3 \eta+\frac{(-3 \eta)^{2}}{2 !}+\frac{(-3 \eta)^{3}}{3 !}+\frac{(-3 \eta)^{4}}{4 !}+\cdots\right)
\end{aligned}
$$

The exact solution in closed form is

$$
\mu(\zeta, \psi, \mathfrak{J}, \eta)=e^{-3 \eta} \sin \zeta \sin \psi \sin \Im
$$

In Figure 7, the exact and the VITM solutions of problem 4 at $\delta=1$ are shown by subgraphs, respectively. From the given figures, it can be seen that both the VITM and exact results are in close contact with each other. Also, in Figure 8, the VITM results of problem 4 are investigated at different fractional-order $\delta=0.8$ and 0.6 . It is analyzed that time-fractional problem results are convergent to an integer order effect as time-fractional analysis to integer order.

4.5. Example. Consider the fractional-order nonlinear convection-diffusion equation

$$
\frac{\partial^{\delta} \mu}{\partial \eta^{\delta}}=\frac{\partial^{2} \mu}{\partial \zeta^{2}}-\frac{\partial \mu}{\partial \zeta}+\frac{\partial}{\partial \eta}\left(\mu \frac{\partial^{2} \mu}{\partial \zeta^{2}}\right)-2 \zeta, 0<\zeta \leq 1,0<\delta \leq 1, \eta>0
$$



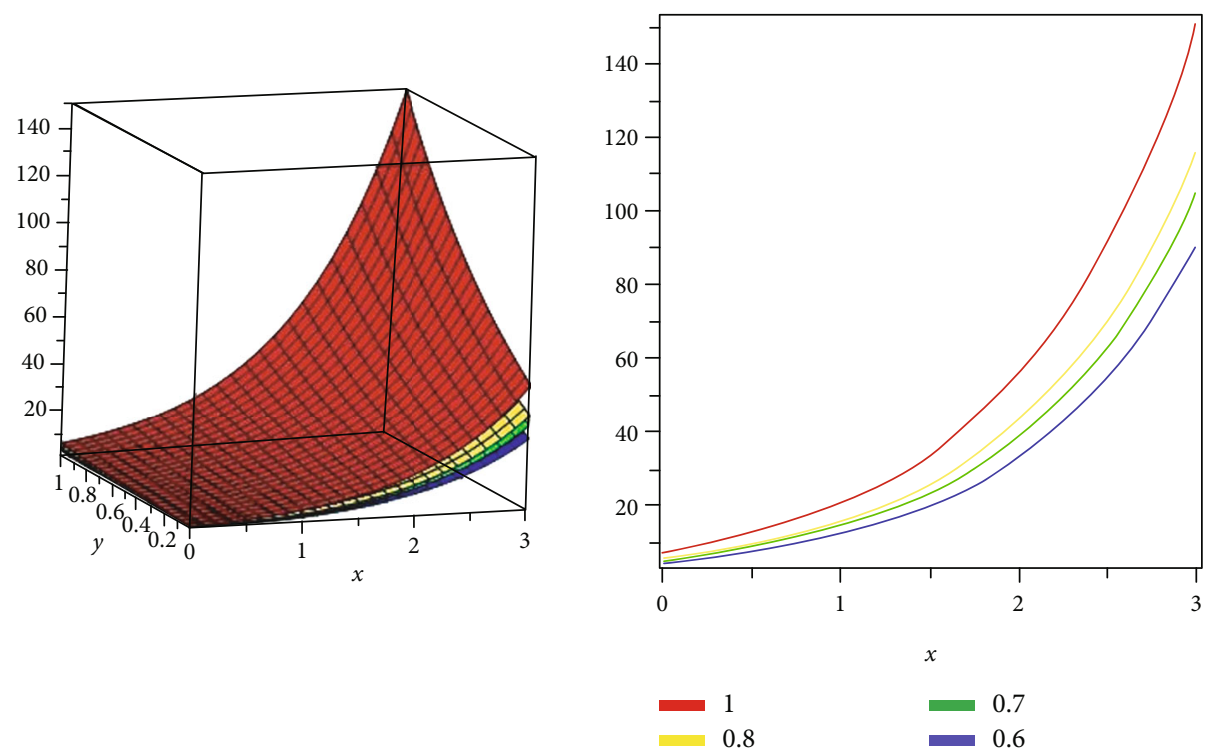

Figure 6: The different fractional-order graphs of $\delta$ Problem 3.3.
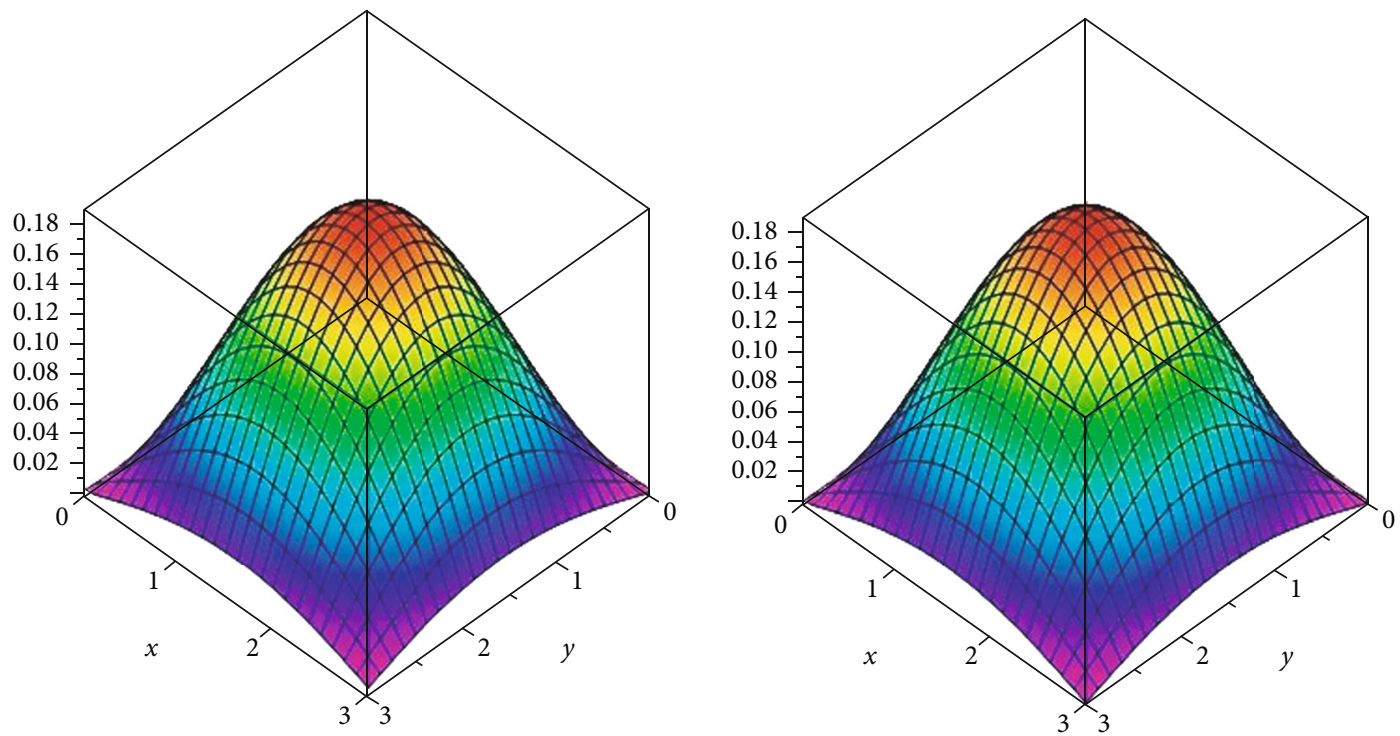

Figure 7: Graph of exact and approximate solutions of Problem 3.4.

with the boundary conditions

$$
\mu(0, \eta)=2 \eta, \mu(1, \eta)=1+2 \eta
$$

and initial condition

$$
\mu(\zeta, 0)=\zeta^{2}
$$

Applying VITM on equation (56), we have

$$
\begin{aligned}
\mu_{m+1}(\zeta, \eta)= & S^{-1}\left[\frac{\mu_{m}(\zeta, \eta)}{s}\right] \\
& +S^{-1}\left[\lambda(s) S\left\{\frac{\partial^{2} \mu_{m}}{\partial \zeta^{2}}-\frac{\partial \mu_{m}}{\partial \zeta}+\frac{\partial}{\partial \eta}\left(\mu_{m} \frac{\partial^{2} \mu_{m}}{\partial \zeta^{2}}\right)-2 \zeta\right\}\right],
\end{aligned}
$$

where the Lagrange multiplier is $\lambda(s)$.

$$
\lambda(s)=-\frac{u^{\delta}}{s^{\delta}},
$$

$$
\begin{aligned}
\mu_{m+1}(\zeta, \eta)= & S^{-1}\left[\frac{\mu_{m}(\zeta, \eta)}{s}\right] \\
& -S^{-1}\left[\frac{u^{\delta}}{s^{\delta}} S\left\{\frac{\partial^{2} \mu_{m}}{\partial \zeta^{2}}-\frac{\partial \mu_{m}}{\partial \zeta}+\frac{\partial}{\partial \eta}\left(\mu_{m} \frac{\partial^{2} \mu_{m}}{\partial \zeta^{2}}\right)-2 \zeta\right\}\right]
\end{aligned}
$$

Now take,

$$
\mu_{0}(\zeta, \eta)=\zeta^{2}
$$



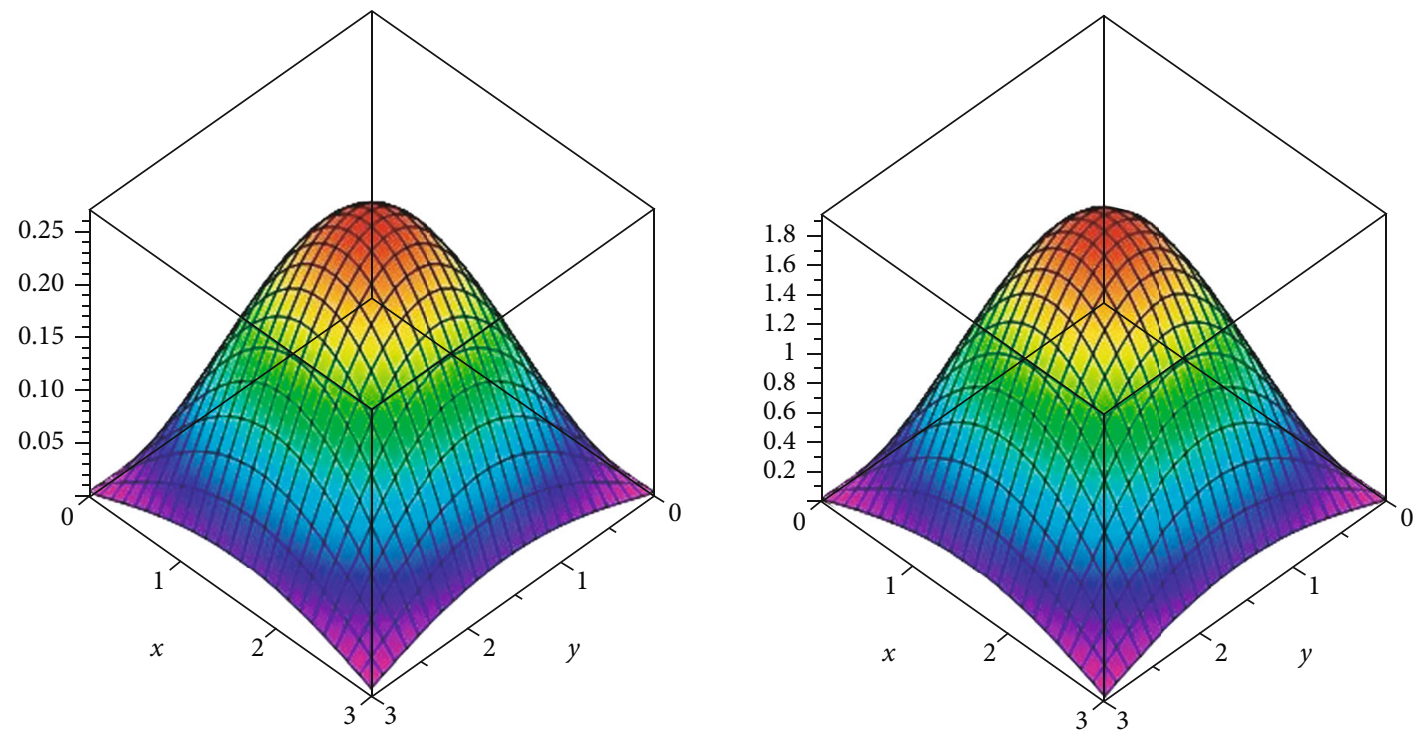

Figure 8: The fractional-order of $\delta=0.8$ and 0.6 of Problem 3.4.

consequently, we get

$$
\begin{aligned}
& m=0,1,2,3 \ldots \\
& \mu_{1}(\zeta, \eta)=S^{-1}\left[\frac{\mu_{0}(\zeta, \eta)}{s}\right] \\
& -S^{-1}\left[\frac{u^{\delta}}{s^{\delta}} S\left\{\frac{\partial^{2} \mu_{0}}{\partial \zeta^{2}}-\frac{\partial \mu_{0}}{\partial \zeta}+\frac{\partial}{\partial \eta}\left(\mu_{0} \frac{\partial^{2} \mu_{0}}{\partial \zeta^{2}}\right)-2 \zeta\right\}\right], \\
& \mu_{1}(\zeta, \eta)=\zeta^{2}+(2-4 \zeta) \frac{\eta^{\delta}}{\Gamma(\delta+1)}, \\
& \mu_{2}(\zeta, \eta)=S^{-1}\left[\frac{\mu_{1}(\zeta, \eta)}{s}\right] \\
& -S^{-1}\left[\frac{u^{\delta}}{s^{\delta}} S\left\{\frac{\partial^{2} \mu_{1}}{\partial \zeta^{2}}-\frac{\partial \mu_{1}}{\partial \zeta}+\frac{\partial}{\partial \eta}\left(\mu_{1} \frac{\partial^{2} \mu_{1}}{\partial \zeta^{2}}\right)-2 \zeta\right\}\right], \\
& \mu_{2}(\zeta, \eta)=\zeta^{2}+(2-4 \zeta) \frac{\eta^{\delta}}{\Gamma(\delta+1)}+4 \frac{\eta^{2 \delta}}{\Gamma(2 \delta+1)} \\
& -4 \zeta(3 \zeta-1) \frac{\eta^{2 \delta-1}}{\Gamma(2 \delta)}, \\
& \mu_{3}(\zeta, \eta)=S^{-1}\left[\frac{\mu_{2}(\zeta, \eta)}{s}\right] \\
& -S^{-1}\left[\frac{u^{\delta}}{s^{\delta}} S\left\{\frac{\partial^{2} \mu_{2}}{\partial \zeta^{2}}-\frac{\partial \mu_{2}}{\partial \zeta}+\frac{\partial}{\partial \eta}\left(\mu_{2} \frac{\partial^{2} \mu_{2}}{\partial \zeta^{2}}\right)-2 \zeta\right\}\right], \\
& \mu_{3}(\zeta, \eta)=\zeta^{2}+(2-4 \zeta) \frac{\eta^{\delta}}{\Gamma(\delta+1)}+4 \frac{\eta^{2 \delta}}{\Gamma(2 \delta+1)} \\
& -4 \zeta(3 \zeta-1) \frac{\eta^{2 \delta-1}}{\Gamma(2 \delta)}-24 \frac{\eta^{3 \delta-1}}{\Gamma(3 \delta)}-4(6 \zeta+1) \frac{\eta^{3 \delta-1}}{\Gamma(3 \delta)} \\
& -4 \zeta^{2}(6 \zeta-1) \frac{\eta^{3 \delta-2}}{\Gamma(3 \delta-1)}-8(1-2 \zeta) \frac{\Gamma(2 \delta+1) \eta^{3 \delta-1}}{\Gamma(3 \delta)(\Gamma(\delta+1))^{2}} \\
& +8 \zeta \frac{\eta^{3 \delta-1}}{\Gamma(3 \delta)}+8 \zeta^{2}(1-3 \zeta) \frac{\eta^{3 \delta-2}}{\Gamma(3 \delta-1)} \text {. }
\end{aligned}
$$

The approximate result of equation (56) can be achieved as

$$
\begin{aligned}
\mu(\zeta, \eta)= & \zeta^{2}+(2-4 \zeta) \frac{\eta^{\delta}}{\Gamma(\delta+1)}+4 \frac{\eta^{2 \delta}}{\Gamma(2 \delta+1)} \\
& -4 \zeta(3 \zeta-1) \frac{\eta^{2 \delta-1}}{\Gamma(2 \delta)}-24 \frac{\eta^{3 \delta-1}}{\Gamma(3 \delta)} \\
& -4(6 \zeta+1) \frac{\eta^{3 \delta-1}}{\Gamma(3 \delta)}-4 \zeta^{2}(6 \zeta-1) \frac{\eta^{3 \delta-2}}{\Gamma(3 \delta-1)} \\
& -8(1-2 \zeta) \frac{\Gamma(2 \delta+1) \eta^{3 \delta-1}}{\Gamma(3 \delta)(\Gamma(\delta+1))^{2}}+8 \zeta \frac{\eta^{3 \delta-1}}{\Gamma(3 \delta)} \\
& +8 \zeta^{2}(1-3 \zeta) \frac{\eta^{3 \delta-2}}{\Gamma(3 \delta-1)}+\cdots
\end{aligned}
$$

The exact result of equation (56) is

$$
\mu(\zeta, \eta)=\zeta^{2}+2 \eta .
$$

\section{Conclusion}

In this article, an extended variational iteration transform method is implemented to achieve the analytical result of time-fractional diffusion equations. The suggested method is an effective and simple tool to solve fractional-order partial differential equations, because it applies the Lagrange multiplier directly to solve fractional-order partial differential equations. In conclusion, the current technique has the small number of calculations and straightforward implementation and therefore can be applied to other fractionalorder partial differential equation, which frequently arises in applied science. 


\section{Abbreviations}

VITM: Variational iteration transform method

ST: $\quad$ Shehu transform

DE: Diffusion equation

FC: $\quad$ Fractional calculus

PDEs: Partial differential equations

ADM: Adomian decomposition method.

\section{Data Availability}

The numerical data used to support the findings of this study are included within the article.

\section{Conflicts of Interest}

The authors declare that they have no conflicts of interest.

\section{Authors' Contributions}

Nehad Ali Shah and Jae Dong Chung contributed equally to this work and are first coauthors.

\section{Acknowledgments}

The authors extend their appreciation to the Deanship of Scientific Research at King Khalid University for funding this work through a research group program under Grant No. RGP.2/38/42. This work was supported by Korea Institute of Energy Technology Evaluation and Planning (KETEP) grant funded by the Korea government (MOTIE) (20202020900060, The Development and Application of Operational Technology in Smart Farm Utilizing Waste Heat from Particulates Reduced Smokestack)

\section{References}

[1] K. B. Oldham and J. Spanier, The Fractional Calculus, Academic Press, New York, 1974.

[2] K. S. Miller and B. Ross, An Introduction to the Fractional Calculus and Fractional Differential Equations, John Wiley and Sons, Inc., New York, 2003.

[3] I. Podlubny, Fractional Differential Equations, Academic Press, New York, 1999.

[4] A. A. Kilbas, H. M. Srivastava, and J. J. Trujillo, Theory and Application of Fractional Differential Equations, Elsevier, Amsterdam, 2006.

[5] A. Bejan, "Second-law analysis in heat transfer and thermal design," in Advances in Heat Transfers, vol. 15, pp. 1-58, Academic Press Inc., 1982.

[6] A. Bejan, "A study of entropy generation in fundamental convective heat transfer," Journal of Heat Transfer, vol. 101, no. 4, pp. 718-725, 1979.

[7] P. Agarwal and A. A. El-Sayed, "Non-standard finite difference and Chebyshev collocation methods for solving fractional diffusion equation," Physica A: Statistical Mechanics and its Applications, vol. 500, pp. 40-49, 2018.

[8] L. Li, Z. Jiang, and Z. Yin, "Fourth-order compact finite difference method for solving two-dimensional convectiondiffusion equation," Advances in Difference Equations, vol. 2018, no. 1, 24 pages, 2018 .
[9] M. Badr, A. Yazdani, and H. Jafari, "Stability of a finite volume element method for the time-fractional advection diffusion equation," Numerical Methods for Partial Differential Equations, vol. 34, no. 5, pp. 1459-1471, 2018.

[10] S. Das, "Analytical solution of a fractional diffusion equation by variational iteration method," Computers \& Mathematics with Applications, vol. 57, no. 3, pp. 483-487, 2009.

[11] S. S. Ray, "Analytical solution for the space fractional diffusion equation by two-step Adomian decomposition method," Communications in Nonlinear Science and Numerical Simulation, vol. 14, no. 4, pp. 1295-1306, 2009.

[12] F. Liu, P. Zhuang, I. Turner, K. Burrage, and V. Anh, “A new fractional finite volume method for solving the fractional diffusion equation," Applied Mathematical Modelling, vol. 38, no. 15-16, pp. 3871-3878, 2014.

[13] M. Inokuti, H. Sekine, and T. Mura, "General use of the Lagrange multiplier in nonlinear mathematical physics," in Variational method in the mechanics of solids, vol. 33, pp. 156-162, Elsevier, 1978.

[14] J.-H. He, “Approximate analytical solution for seepage flow with fractional derivatives in porous media," Computer Methods in Applied Mechanics and Engineering, vol. 167, no. 1-2, pp. 57-68, 1998.

[15] J.-H. He, "Variational iteration method - a kind of non-linear analytical technique: some examples," International Journal of Non-Linear Mechanics, vol. 34, no. 4, pp. 699-708, 1999.

[16] J. Hristov, “An exercise with the He's variation iteration method to a fractional Bernoulli equation arising in transient conduction with non-linear heat flux at the boundary," International Review of Chemical Engineering, vol. 4, no. 5, pp. 489-497, 2012.

[17] E. Hetmaniok, K. Kaczmarek, D. Słota, R. Wituła, and A. Zielonka, "Application of the variational iteration method for determining the temperature in the heterogeneous casting-mould system," International Review of Chemical Engineering, vol. 4, no. 5, pp. 511-515, 2012.

[18] M. A. Abdou and A. A. Soliman, "Variational iteration method for solving Burger's and coupled Burger's equations," Journal of Computational and Applied Mathematics, vol. 181, no. 2, pp. 245-251, 2005.

[19] A. M. Wazwaz, "The variational iteration method: a reliable analytic tool for solving linear and nonlinear wave equations," Computers and Mathematics with Applications, vol. 54, no. 78, pp. 926-932, 2007.

[20] M. Inc, "Numerical simulation of $\mathrm{KdV}$ and $\mathrm{mKdV}$ equations with initial conditions by the variational iteration method," Chaos, Solitons and Fractals, vol. 34, no. 4, pp. 1075-1081, 2007.

[21] J. H. He and X. H. Wu, "Variational iteration method: new development and applications," Computers and Mathematics with Applications, vol. 54, no. 7-8, pp. 881-894, 2007.

[22] G. C. Wu and E. W. M. Lee, "Fractional variational iteration method and its application," Physics Letters A, vol. 374, no. 25, pp. 2506-2509, 2010.

[23] E. Hesameddini and H. Latifizadeh, "Reconstruction of variational iteration algorithms using the Laplace transform," International Journal of Nonlinear Sciences and Numerical Simulation, vol. 10, no. 11-12, pp. 1377-1382, 2009.

[24] S. A. Khuri and A. Sayfy, "A Laplace variational iteration strategy for the solution of differential equations," Applied Mathematics Letters, vol. 25, no. 12, pp. 2298-2305, 2012. 
[25] G. C. Wu and D. Baleanu, "Variational iteration method for fractional calculus an universal approach by Laplace transform," Advances in Difference Equations, vol. 2013, 9 pages, 2013.

[26] H. Jafari and H. K. Jassim, "Local fractional Laplace variational iteration method for solving nonlinear partial differential equations on Cantor sets within local fractional operators," Journal of Zankoy Sulaimani - Part A, vol. 16, pp. 49-57, 2014.

[27] H. F. Ahmed, M. S. M. Bahgat, and M. Zaki, "Numerical approaches to system of fractional partial differential equations," Journal of the Egyptian Mathematical Society, vol. 25, no. 2, pp. 141-150, 2017.

[28] J. A. T. Machado, D. Baleanu, W. Chen, and J. Sabatier, "New trends in fractional dynamics," Journal of Vibration and Control, vol. 20, no. 7, pp. 963-963, 2014.

[29] D. Baleanu, Z. B. Güvenç, and J. A. Tenreiro Machado, Eds., New Trends in Nanotechnology and Fractional Calculus Applications, Springer, New York, 2010.

[30] S. Maitama and W. Zhao, "New integral transform: Shehu transform a generalization of Sumudu and Laplace transform for solving differential equations," 2019, https://arxiv.org/ abs/1904.11370.

[31] A. Bokhari, D. Baleanu, and R. Belgacem, "Application of Shehu transform to Atangana-Baleanu derivatives," Journal of Mathematics and Computer Science, vol. 20, no. 2, pp. 101-107, 2019.

[32] R. Belgacem, D. Baleanu, and A. Bokhari, "Shehu transform and applications to Caputo-fractional differential equations," International Journal of Analysis and Applications, vol. 17, no. 6, pp. 917-927, 2019. 